\title{
Photoionization Yield Spectra below the Atomic Ionization Limit in Xenon
}

\author{
R. Reininger and V. Saile \\ Hamburger Synchrotronstrahlungslabor, Deutsches Elektronen-Synchrotron, \\ D-2000 Hamburg 52, Germany \\ and \\ P. Laporte \\ Equipe de Spectroscopie de Centre National de la Recherche Scientifique, \\ F-42023 Saint Etienne Cedex, France
}

(Received 10 December 1984)

\begin{abstract}
Photocurrent yield and absorption spectra for xenon were measured as functions of pressure $\left(p \leqslant 1 \times 10^{5} \mathrm{~Pa}\right)$ between $\sim 11.0$ and $11.8 \mathrm{eV}$. With a pressure-independent yield function $Y(h \nu)$ the photocurrent spectra can be calculated from the absorption spectra with the assumption of a "dead layer" at the surface of the LiF window. $Y(h v)$ is roughly proportional to the number of atomic transitions with excitation energies between $11.10 \mathrm{eV}$ and $h \nu$. This behavior can be explained with a simple statistical model for the decay of the Rydberg states.
\end{abstract}

PACS numbers: $32.80 . \mathrm{Fb}, 33.80 . \mathrm{Eh}$

Rare gases are ideally suited to study basic processes in atomic and molecular systems. This includes Rydberg states, autoionization, dimer and excimer formation, and photoionization. A detailed knowledge of electronic excitation and the relaxation phenomena is of considerable theoretical interest but also has implications for the technical applications of rare gases, i.e., in excimer lasers and detectors. The ionization of rare gases has been studied recently by use of supersonic beams ${ }^{1-3}$ and by multiphoton excitation with lasers. ${ }^{4}$ Rare gases exhibit strong pressure effects in both fluorescence and ionization. Even at pressures as low as $40 \mathrm{~Pa}$ photoionization has been observed at photon energies that are considerably lower than the first atomic ionization potential of the rare-gas atoms. ${ }^{5,6}$ The aim of this Letter is to present a detailed experimental investigation of the photoionization in $\mathrm{Xe}$, its pressure dependence, and its relationship to Rydberg states. The results might be of interest for various fields ranging from multiphoton excitation experiments ${ }^{7,8}$ to rare-gas detectors. ${ }^{9}$

Rare gases can be ionized with photon energies below the atomic ionization potential but above an energy close to the adiabatic ionization potential of the rare-gas dimer..$^{5,6,10}$ For low pressures $p \leqslant 1.5 \times 10^{3}$ $\mathrm{Pa}$ peaks in the photocurrent spectra have been observed which coincide with the well-known atomic transitions. This was explained by the formation of excited rare-gas dimers $R_{2}^{*}$ and their decay into a dimer ion $R_{2}^{+}$and an electron. The minimum photon energy required for this process is very close to the adiabatic ionization energy $I_{d}$ of the rare-gas molecule. With increasing pressure the lines in the photocurrent spectra broaden and finally $\left(p \geqslant 4 \times 10^{4} \mathrm{~Pa}\right)$ the wings of the lines overlap to form a continuum but with deep dips around the position of the atomic lines. ${ }^{10}$

The spectral dependence of the photocurrent and the absorption was recorded with synchrotron radiation from the storage ring DORIS at DESY, Hamburg. The light was monochromatized with a $3-\mathrm{m}$ normalincidence monochromator ${ }^{11}$ working for this experiment at an energy resolution of approximately $6 \mathrm{meV}$. The $\mathrm{Xe}$ pressure ranged between $7.3 \times 10^{2} \mathrm{~Pa}$ to $9.7 \times 10^{4} \mathrm{~Pa}$. In the gas cell two 10 -mm-long stainlesssteel platelets separated by $2 \mathrm{~mm}$ and parallel to the direction of the incoming light (beam size $1 \times 1 \mathrm{~mm}^{2}$ ) served as electrodes. The distance between the front end of the platelets and the $\mathrm{LiF}$ window was less than $1 \mathrm{~mm}$. The applied voltage was chosen to be high enough to ensure current saturation.

In order to obtain the absolute number of carriers created per photon absorbed in the Xe gas, the photoelectric yield of an $\mathrm{Al}$ film mounted behind the front $\mathrm{LiF}$ window of the cell was measured. With the absolute yield of Al given by Cairns and Samson ${ }^{12}$ and by comparison of the photocurrents of $\mathrm{Al}$ and $\mathrm{Xe}$ an absolute scale for the photocurrent yield spectra was established.

The transmission experiments were performed with three different cells with absorption lengths of 11.5 $\mathrm{mm}, 2.1 \mathrm{~mm}$, and $7 \pm 1 \mu \mathrm{m}$ between the two LiF windows. The $7-\mu \mathrm{m}$ cell was used only for the absorption spectrum at the highest pressure and its length was determined as described by Laporte, Subtil, and Castex. ${ }^{13}$

Representative spectra for five pressures are shown in Fig. 1 together with the positions of dipole-allowed atomic transitions. For the lowest pressures narrow peaks at the positions of the atomic lines are observed. The line shape and full width at half maximum are essentially given by the monochromator with a resolution of $6 \mathrm{meV}$. At higher pressures the lines undergo asymmetric broadening, expanding more to the lower energies. There is also some growth in the heights of the peaks. A further increase in pressure reveals the appearance of dips at the position of the atomic transi- 


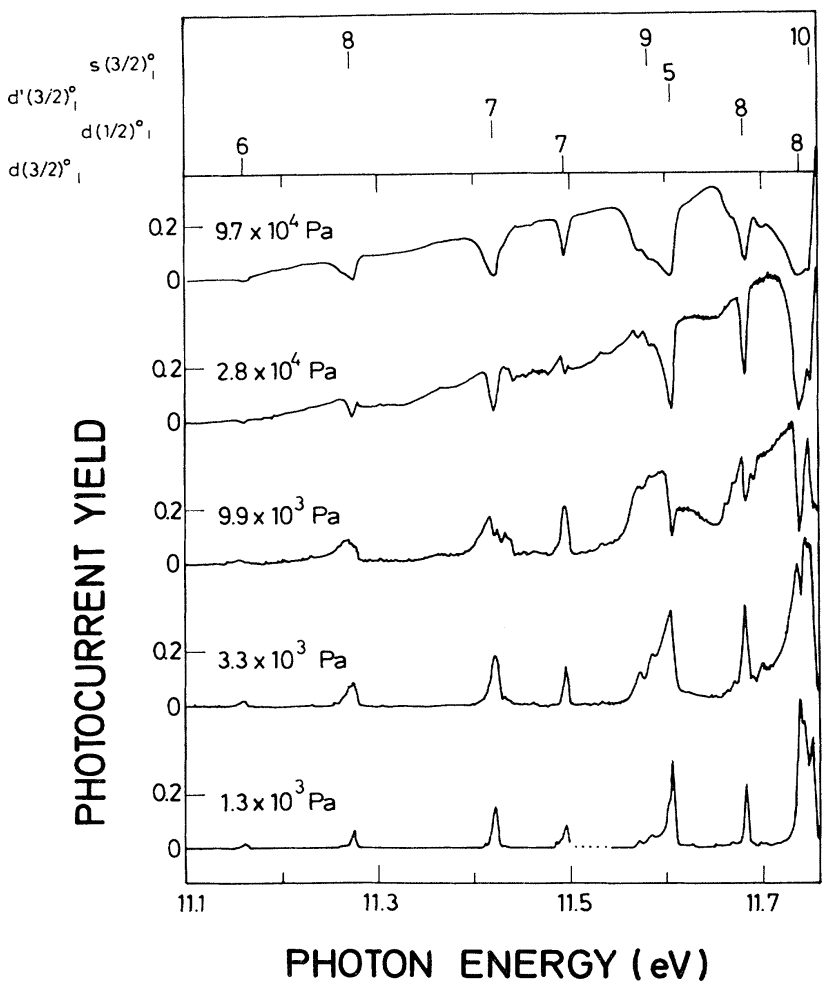

FIG. 1. Photocurrent yield in units of electrons (or ions) per absorbed photon for five different Xe densities. In the uppermost part the dipole-allowed atomic transitions are indicated.

tions. These dips become deeper and broader with increasing pressure. ${ }^{10}$ The wings of the lines overlap, forming finally an envelope for the photocurrent spectra.

As mentioned above, absorption spectra were measured for the same pressures as the photoionization yield spectra. Figure 2 shows the absorption coefficient $k$ for three different pressures. Again at low pressures the spectral resolution was insufficient to measure the intrinsic width and height of the extremely sharp lines. For higher pressures (curves $b$ and $c$ in Fig. 2) the lines broaden mainly in the low-energy wing and exhibit on top of both wings new absorption features. Comparing Figs. 1 and 2 shows that even these new bands are reproduced in the photocurrent spectra. Most of them can be attributed to molecular bands originating from dipole-forbidden transitions in the free atom.

Photoionization of rare-gas dimers was studied in supersonic beam experiments. ${ }^{1-3}$ In these experiments molecules with interatomic distances given by the shallow van der Waals minimum in the groundstate potential are excited by vacuum ultraviolet light. Then photoionization is possible only for $h \nu \geqslant I_{d}$ $=11.127 \mathrm{eV},{ }^{1}$ where $I_{d}$ is the adiabatic ionization potential of the rare-gas molecule. In the photoconduc-

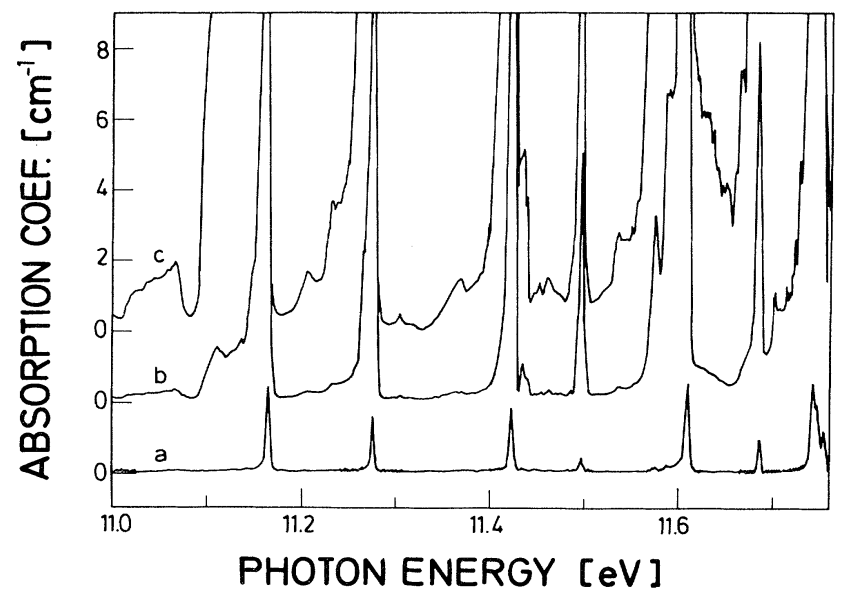

FIG. 2. Absorption spectra for three representative pressures: curve $a, 1.3 \times 10^{3} \mathrm{~Pa}$; curve $b, 9.9 \times 10^{3} \mathrm{~Pa}$; curve $c$ $2.8 \times 10^{4} \mathrm{~Pa}$.

tion experiments described in this Letter one deals with pairs of atoms with distances given by the thermodynamic conditions and the ground-state potential. $^{14}$ In this case we expect and observe (see Fig. 1 and Ref. 10) a threshold for photoconduction at $h \nu$ $\sim I_{d}-I_{\text {diss }}=11.127 \mathrm{eV}-0.023 \mathrm{eV}=11.104 \mathrm{eV}$ where $I_{\text {diss }}{ }^{15}$ is the dissociation energy of the $\mathrm{Xe}_{2}$ molecule in its ground state.

The first step in a series of eventually quite complicated processes leading to photoionization is the absorption of a photon. Comparing Figs. 1 and 2 one can easily realize that in the wings of the atomic lines the photocurrent increases with increasing pressure, i.e., with increasing absorption. For the line center a peak is observed at low pressures and at higher pressures a dip which broadens upon a further increase of pressure. Such a behavior can be explained if we assume that the photocurrent follows the absorption coefficient only up to a certain limit. In other words, if the penetration depth of the light becomes too small the photocurrent decreases. This is the case if carriers produced close to the inner surface of the window do not contribute to the photocurrent measured. Such a dead layer could be due to several reasons, e.g., an efficient recombination process of electrons and ions induced by the window, or a reduced or distorted electric field close to the window diminishing the collection efficiency for the carriers. ${ }^{16}$ In fact, the depth of the minimum is reduced with increasing electric field strength, but even with fields close to an electric breakdown in the gas dips are still observed.

The number of photons per second contributing to the photocurrent is given by

$$
I=I_{0}\left[\exp \left(-k x_{0}\right)-\exp (-k l)\right]
$$

for a photon intensity $I_{0}$ transmitted by the entrance window, an absorption coefficient $k$, and the length 
$l=10 \mathrm{~mm}$ of the cell. The first term in Eq. (1) accounts for the dead layer with a depth $x_{0}$. For the ionization process the various possible decay channels are represented for this density range by a pressureindependent yield function $Y(h \nu)$. The photocurrent $i(h \nu, p)$ is given then by

$$
i(h \nu, p)=Y I_{0}\left[\exp \left(-k x_{0}\right)-\exp (-k l)\right] .
$$

From the experimental results the yield function $Y$ for photoionization is obtained in the following way: The photocurrent spectra at higher pressures in Fig. 1 led us to the assumption that $Y$, i.e., the envelope function for the photocurrent neglecting the dips, should be of the form

$$
Y=a(h \nu-11.10 \mathrm{eV})+b(h \nu-11.10 \mathrm{eV})^{2},
$$

with $11.10 \mathrm{eV} \leqslant h \nu \leqslant 12.13 \mathrm{eV}$, where $11.10 \mathrm{eV}$ is the threshold for photoionization as discussed above. Fitting the experimental photocurrent spectra for five pressures by Eq. (2), with the measured $k$ values, $a$ and $b$ were found to be 0.31 and 0.68 , respectively. The resulting pressure-independent yield function $Y(h \nu)$ is shown in Fig. 3.

The physical interpretation of the photon energy dependence of $Y(h \nu)$ is also given in Fig. 3. After excitation the electronic state relaxes-following a more or less complicated pattern - to the lowest excited state of the atom or dimer. The probability $Y(h \nu)$ that a dimer ion and an electron can be formed during the relaxation process should depend on the number of states between the adiabatic ionization potential $11.10 \mathrm{eV}$ of the dimer and the excitation energy $h \nu$. Lacking detailed molecular potential curves for this energy range we added for a photon energy $h \nu$ the number of all atomic states ${ }^{17}$ between $11.10 \mathrm{eV}$ and $h \nu$

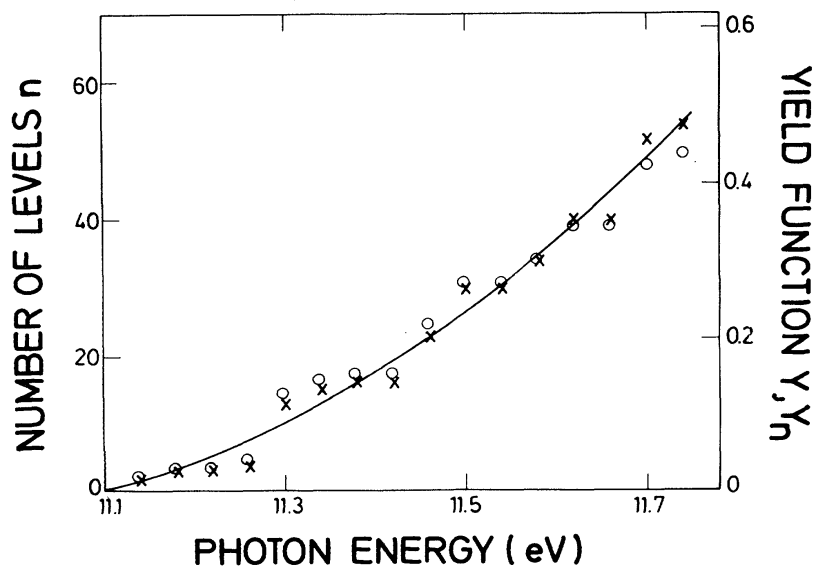

FIG. 3. Crosses, number of atomic levels above $11.10 \mathrm{eV}$ from Ref. 17. Circles, $Y_{n}$ according to Eq. (4). The solid line represents the experimentally determined yield function in units of electrons or ions per absorbed photon. (crosses in Fig. 3). If we assume that each of these Rydberg states can decay either to an electron-ion pair with a probability $Y_{1}$ or to the next lower Rydberg state with a probability $\left(1-Y_{1}\right)$, the yield obtained from the $n$th state above $11.10 \mathrm{eV}$ should be

$$
Y_{n}=1-\left(1-Y_{1}\right)^{n} \text {. }
$$

The circles in Fig. 3 represent the values for $Y_{n}$ using $Y_{1}=1.05 \%$. Even with this oversimplified model the agreement between $Y_{n}$ and the experimental yield function $Y(h v)$ is convincing. $Y_{n}$ converges to 1 at the first atomic ionization limit of $\mathrm{Xe}$ at $12.13 \mathrm{eV}$. On the other hand an extrapolation of the experimental yield function $Y(h v)$ gives 1.04 for the same limit.

With the yield function $Y$, the whole photocurrent spectra for all pressures measured were reproduced by application of Eq. (2), with the dead-layer thickness $x_{0}$ as the only free parameter. The results for three representative pressures are shown in Fig. 4. For the lowest pressure $1.3 \times 10^{3} \mathrm{~Pa}$ the agreement between measured and calculated spectrum is excellent without any dead layer. At the highest pressure $9.7 \times 10^{4} \mathrm{~Pa}$ the dashed line was calculated with $x_{0}=20 \mu \mathrm{m}$. The agreement between photocurrent spectra and fit is convincing.

The main conclusion of the present study is, that in the spectral range between the adiabatic ionization limit of the dimer and the atomic ionization limit, the photoionization can be described by the absorption coefficient and the pressure-independent yield func-

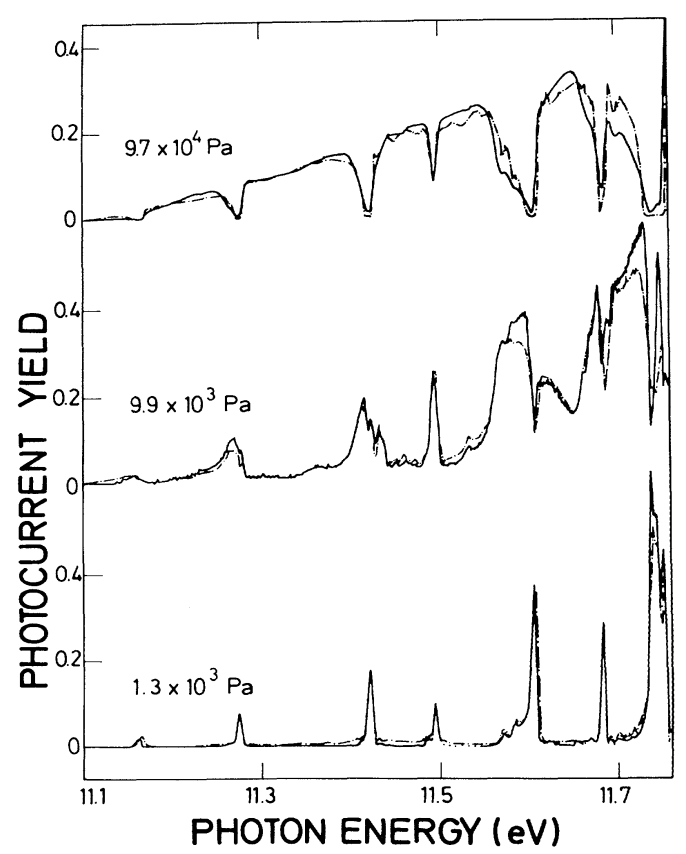

FIG. 4. Experimental (solid lines) and calculated (dotdashed lines) photocurrent spectra according to Eq. (2). 
tion only. Preliminary results show that this concept can be also applied to $\mathrm{Kr}$ and $\mathrm{Ar}$. $\mathrm{Ne}$ and $\mathrm{He}$ might behave differently and experiments to clarify this question are underway. The implications of the yield function on the excimer fluorescence from Xe and the competition between the collection of charges and the fluorescence decay are discussed elsewhere. ${ }^{18}$

We want to thank Professor I. T. Steinberger, Hebrew University of Jerusalem, for numerous discussions, his help, and criticism.

1C. Y. Ng, D. J. Trevor, B. H. Mahan, and Y. T. Lee, J. Chem. Phys. 65, 4327 (1976).

2S. T. Pratt and P. M. Dehmer, Chem. Phys. Lett. 87, 533 (1982).

3P. M. Dehmer, J. Chem. Phys. 76, 1263 (1982).

${ }^{4}$ A. W. McCown, M. N. Ediger, and J. G. Eden, Phys. Rev. A 29, 2611 (1984).

5R. E. Huffmann and D. H. Katayama, J. Chem. Phys. 45, 138 (1966).

6J. A. R. Samson and R. B. Cairns, J. Opt. Soc. Am. 56, 1140 (1966).

7J. C. Miller, R. N. Compton, M. G. Payne, and W. W.
Garrett, Phys. Rev. Lett. 45, 114 (1980).

8J. Glownia and R. K. Sander, Phys. Rev. Lett. 49, 21 (1982).

${ }^{9}$ M. Suzuki, J. Ruan(Gen), and S. Kubota, Nucl. Instrum. Methods 192, 565 (1982).

10P. Laporte, V. Saile, R. Reininger, U. Asaf, and I. T. Steinberger, Phys. Rev. A 28, 3613 (1983).

11 V. Saile, P. Gürtler, E. E. Koch, A. Kozevnikov, M. Skibowski, and W. Steinmann, Appl. Opt. 15, 2559 (1976).

${ }_{12}$ R. B. Cairns and J. A. R. Samson, J. Opt. Soc. 56, 1568 (1966).

13P. Laporte, J. L. Subtil, and M. C. Castex, in Spectral Line Shapes (Walter de Gruyter, Berlin, 1983), Vol. 2, p. 583.

${ }^{14}$ M. C. Castex, J. Chem. Phys. 74, 759 (1981).

${ }^{15}$ D. E. Freeman, K. Yoshino, and Y. Tanaka, J. Chem. Phys. 61, 4880 (1974).

16In Ref. 10 we suggested that the dips might result from collisional dissociation of the excimers which is most effective in the line center. The good fit of Fig. 4 makes this assumption superfluous.

${ }^{17}$ C. E. Moore, U. S. National Bureau of Standards Circulation No. 467 (U.S. GPO, Washington, D.C., 1958), Vol. 3, p. 113.

${ }^{18 V}$. Saile, R. Reininger, A. M. Köhler, and G. L. Findley, to be published. 